\title{
Viscous slip coefficients for binary gas mixtures measured from mass flow rates through a single microtube
}

\author{
H. Yamaguchi, ${ }^{1, a)}$ K. Takamori, ${ }^{1)}$ P. Perrier, ${ }^{2)}$ I. Graur, ${ }^{2)}$ Y. Matsuda, ${ }^{3)}$ and T. \\ Niimi ${ }^{1)}$ \\ ${ }^{1}$ Department of Micro-Nano Systems Engineering, Nagoya University, Nagoya, Aichi, 464-8603, Japan \\ ${ }^{2}$ IUSTI UMR 7343, Aix-Marseille Université, Marseille, 13453, France \\ ${ }^{3}$ Institute of Materials and Systems for Sustainability, Nagoya University, Nagoya, Aichi, 464-8603, Japan
}

The viscous slip coefficient for helium-argon binary gas mixture is extracted from the experimental values of the mass flow rate through a microtube. The mass flow rate is measured by the constant-volume method. The viscous slip coefficient was obtained by identifying the measured mass flow rate through a microtube with the corresponding analytical expression, which is as a function of the Knudsen number. The measurements were carried out in the slip flow regime where the firstorder slip boundary condition can be applied. The measured viscous slip coefficients of binary gas mixtures exhibit a concave function of the molar ratio of the mixture, showing a similar profile with numerical results. However, from the detailed comparison between the measured and numerical values with the complete and incomplete accommodation at a surface, it is inappropriate to estimate the viscous slip coefficient for the mixture numerically by employing separately measured tangential momentum accommodation coefficient for each component. The time variation of the molar ratio in the downstream chamber was measured by sampling the gas from the chamber using the quadrupole mass spectrometer. In our measurements, it is indicated that the volume flow rate of argon is larger than that of helium because of the difference in the tangential momentum accommodation coefficient.

\section{INTRODUCTION}

Recently micro gaseous flows through a channel or tube has been extensively studied ${ }^{1-5)}$ along with the development of the micro- and nano-technologies. At micro scale, the Knudsen number, which is a ratio of the mean free path of molecules to the characteristic length of a system, is large, so the number of the molecule-surface collisions is comparable with the molecule-molecule collisions. In addition, the surface-to-volume ratio of a fluid inside the system is also large; thus, the boundary condition at surfaces is important to precisely simulate the flow field. In relatively high Knudsen number flows, the gas-surface interaction is often represented by a single parameter derived from the statistical analysis, which is called the accommodation coefficient ${ }^{6)}$. Under isothermal flow conditions, the flow rate through a microsystem is affected by the exchange of the tangential component of momentums between gas molecules and a surface; therefore, the tangential momentum accommodation coefficient (TMAC) $\alpha_{t}$ is employed to characterize the gas-surface interaction, which is defined by

a) Electronic mail: hiroki@nagoya-u.jp 


$$
\alpha_{t}=\frac{\tau_{\mathrm{i}}-\tau_{\mathrm{r}}}{\tau_{\mathrm{i}}}
$$

where $\tau$ is the mean value of the tangential component of gas molecules' momentum and the indexes $i$ and $r$ represent incident and reflected molecules, respectively.

In the slip flow regime, the flow can be simulated by the Navier-Stokes equations with the slip boundary condition, which is expressed by using the viscous slip coefficient $\sigma_{p}$ as ${ }^{7,3)}$,

$$
u_{x}=A_{1} \lambda \frac{d u_{x}}{d y}, \quad A_{1}=\frac{\sigma_{p}}{k_{\lambda}}
$$

where a solid surface coincide with an $x$-z plane, $x$ is the flow direction, $u_{x}$ is $x$-component of the flow velocity, and $y$ is the axis normal to the surface, respectively. The viscosity coefficient $\mu$ of a single gas at temperature $T$ is calculated by employing the variable hard sphere (VHS) model $^{8)}$

$$
\mu=\mu_{\text {ref }}\left(\frac{T}{T_{\text {ref }}}\right)^{\omega}
$$

where $\mu_{\text {ref }}$ is the viscosity at $T_{\text {ref }}=273.15 \mathrm{~K}$, and $\omega$ is the viscosity index. In the VHS model, $k_{\lambda}$ is expressed by the viscosity index $\omega$ as $k_{\lambda}=\frac{(7-2 \omega)(5-2 \omega)}{15 \sqrt{\pi}}$. The mean free path $\lambda$ is defined by

$$
\lambda=k_{\lambda} \frac{\mu}{p} c_{m}, \quad c_{m}=\sqrt{2 R T}
$$

where $p$ is pressure, $c_{m}$ is the most probable molecular speed, $k$ is the Boltzmann constant, $T$ is temperature, $R$ is the specific gas constant, respectively.

From the kinetic simulation of the slip flow regime, it was found that the viscous slip coefficient can be related to TMAC 3,5, 9 -12); therefore, the viscous slip coefficient can be considered as another way to represent TMAC in a framework of Navier-Stokes equations in the slip flow regime. The viscous slip coefficient and TMAC are very important to characterize the flow field precisely at relatively high Knudsen number flows. The both coefficients have been investigated by many researchers ${ }^{1-5)}$. However, in most of these studies, the authors measured the values of the viscous slip coefficient and TMAC for single gases, while gaseous mixtures are more often employed in practice. The numerical data on the slip coefficient for various mixtures were reported by several researchers ${ }^{9-12}$. On the other hand, there are a few experimental studies. The mass 
flow rate for a gas mixture was measure ${ }^{13)}$ for several values of pressures, but only for a single value of the molar ratio and the viscous slip coefficient was not extracted. Only one data, to the authors' best knowledge, on the viscous slip coefficient for gas mixtures as a function of the molar ratio, measured by using a spinning rotor gauge, was reported ${ }^{14)}$. Nonetheless, there were quantitative and even qualitative discrepancies between the measured and numerically simulated viscous slip coefficients $^{14)}$. Therefore, it is important to investigate experimentally the effect of mixture nature and composition on the viscous slip coefficient and TMAC.

In this study, the viscous slip coefficients of helium-argon binary gas mixtures are obtained from the measured mass flow rate through a microtube. This experimental approach ${ }^{15)}$, which is different from that implemented in Ref. 14, was previously used to determine the viscous slip coefficient and TMAC for single gases. Here, this technique is applied for the first time to measure the viscous slip coefficient of He-Ar mixture as a function of the molar ratio. He-Ar mixtures of five different molar ratios were employed to investigate the effect of mixture composition on the viscous slip coefficient. The time variation of the molar ratio due to the flow from upstream to downstream chambers is measured to investigate the difference in the mass flow rate for each component.

\section{Measurement}

\section{A. Experimental Procedure}

The viscosity slip coefficient was extracted from the measured mass flow rate through a single microtube connecting two chambers initially at different pressures but at the same mixture composition. The measurements were carried out in the slip flow regime. The measurement principle and setup is explained in details in Ref. 15 and a schematic of the setup is shown in

Fig. 1. Two chambers made by SUS304 were connected by a single microtube. The constant-volume method ${ }^{15-17)}$, where the mass flow rate is related to the pressure increase rate in the downstream chamber, was employed for measuring the mass flow rate. From the time derivative of the equation of state for an ideal gas under the constant temperature and volume conditions, the variation of mass in time $\dot{M}$ can be related to the pressure variation rate as,

$$
\dot{M}=\frac{V}{R T} \frac{d p}{d t},
$$

where $V, T, p$ is the volume, temperature, pressure of the downstream chamber, respectively. Pressure of each chamber was measured by temperature controlled capacitance manometers (MKS Baratron® 627B Series). A microtube with a circular cross-section made by fused silica with the inner diameter $D=250 \mu \mathrm{m}$ was used. 


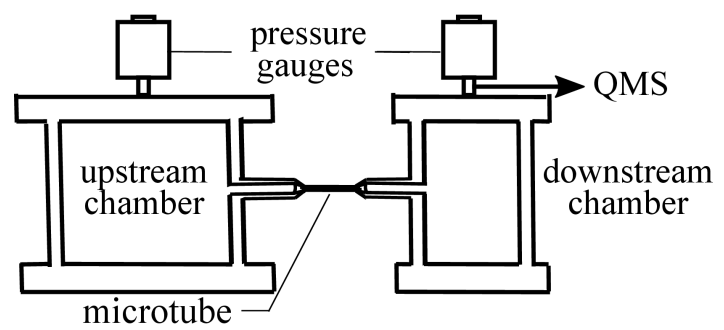

FIG. 1. A cross-sectional schematic of the experimental setup.

The binary mixtures of helium and argon for five different volume (or molar) fractions of $(\mathrm{He}, \mathrm{Ar})=(0 \%, 100 \%),(25 \%$, $75 \%),(50 \%, 50 \%),(75 \%, 25 \%),(100 \%, 0 \%)$, were employed as test gases. These mixtures were commercially obtained (Japan Fine Products). To check the fraction of each component and other residual gases, the gas in the downstream chamber was sampled through a variable leak valve and analyzed by a quadrupole mass spectrometer (Pfeiffer Vacuum, QMS 200) with a pumping station before and after each measurement of the mass flow rate. Due to the connection to the QMS, the volume of the downstream chamber was a little increased from the previously reported value ${ }^{15)}$ to $V_{\mathrm{o}}\left[\mathrm{cm}^{3}\right]=450.26+0.017$ $p_{\mathrm{o}}[\mathrm{Pa}]$, where $p_{\mathrm{o}}$ is the pressure at the downstream chamber.

\section{B. Extraction of the viscous slip coefficient}

The analytical mass flow rate through a tube in the slip flow regime can be obtained by solving the Navier-Stokes equation with the slip boundary condition ${ }^{7,15-17)}$. The flow regime inside the microtube could be characterized by the mean Knudsen number based on the mean pressure $p_{\mathrm{m}}$ between the upstream and downstream chambers, $K n_{\mathrm{m}}=\lambda / D$, where the mean free path is defined by eq. (4). The mean Knudsen number was limited to be below 0.1 ; which corresponds to the slip flow regime. Therefore, the first order slip boundary condition, eq. (2), was applied, and the analytical mass flow rate is expressed as,

$$
\dot{M}=\dot{M}_{\mathrm{C}}\left(1+8 A_{1} K n_{\mathrm{m}}\right), \quad \dot{M}_{\mathrm{C}}=\frac{\pi D^{4} \Delta P P_{\mathrm{m}}}{128 \mu R T L}
$$

where $\dot{M}_{\mathrm{C}}$ is the mass flow rate at the continuum limit $\left(K n_{\mathrm{m}} \rightarrow 0\right)$, namely the Poiseuille mass flow rate, $\Delta P$ is the pressure difference between the upstream and downstream chambers, and $L$ is the length of the microtube, respectively. By dividing eq. (6) by the Poiseuille mass flow rate $\dot{M}_{\mathrm{C}}$, we obtain dimensionless form of the mass flow rate as a function of the mean 
Knudsen number. Thus, we can derive the viscous slip coefficient by fitting the dimensionless mass flow rate as a linear function of the mean Knudsen number and extract then the viscous slip coefficient through the coefficient $A_{1}$ from eq. (2).

\section{Viscosity coefficient for binary gas mixture}

To obtain the viscous slip coefficient, the viscosity coefficient for each gaseous mixture is used in eq. (6). We employed the Wilke's method ${ }^{18)}$ to calculate the viscosity coefficient of a mixture $\mu_{\mathrm{m}}$,

$$
\mu_{\mathrm{m}}=\frac{y_{1} \mu_{1}}{y_{1}+y_{2} \phi_{12}}+\frac{y_{2} \mu_{2}}{y_{2}+y_{1} \phi_{21}}, \quad \phi_{i j}=\frac{\left[1+\left(\frac{\mu_{i}}{\mu_{j}}\right)^{\frac{1}{2}}\left(\frac{m_{j}}{m_{i}}\right)^{\frac{1}{4}}\right]^{2}}{\left[8\left(1+\frac{m_{i}}{m_{j}}\right)\right]^{\frac{1}{2}}},
$$

where $y_{i}, m_{i}$ is the molar fraction and the molecular weight of $i$ component, respectively. The viscosity index of a mixture was calculated from the gradient of a log-log plot for the values of temperature varying from $273.15 \mathrm{~K}$ to $546.15 \mathrm{~K}$, using eqs. (3) and (7). The plots were very well fitted by liner functions, validating that eq. (3) could be applied even for mixtures. The reference viscosity coefficient and viscosity index calculated for the mixtures are listed in Table I.

TABLE I. Viscosity coefficient and index for mixture.

\begin{tabular}{cccccc}
\hline \hline Case & 1 & \multicolumn{1}{c}{2} & \multicolumn{1}{c}{3} & \multicolumn{1}{c}{4} & \multicolumn{1}{c}{5} \\
\hline $\mathrm{He}$ & $0 \%$ & $25 \%$ & $50 \%$ & $75 \%$ & $100 \%$ \\
$\mathrm{Ar}$ & $100 \%$ & $75 \%$ & $50 \%$ & $25 \%$ & $0 \%$ \\
\hline$\mu_{\text {ref }}\left[10^{5} \mathrm{~Pa} \cdot \mathrm{s}\right]$ & 2.117 & 2.168 & 2.212 & 2.200 & 1.865 \\
$\omega$ & 0.81 & 0.80 & 0.78 & 0.75 & 0.66 \\
\hline \hline
\end{tabular}

\section{VISCOUS SLIP COEFFICIENT FOR BINARY GAS MIXTURE}

\section{A. Experimental values}

The inner diameter of the tube was estimated from the measured mass flow rate at the continuum limit $\dot{M}_{\mathrm{C}}$ by using eq. (6), and the error in the estimation of the inner diameter was evaluated by the $95 \%$ confidence interval ${ }^{15)}$. The obtained diameter was $D=252.5 \pm 0.2 \mu \mathrm{m}$ and it was close to the nominal value, indicating the validity of the measurements. The length of the micro tube was $L=57.7 \pm 0.1 \mathrm{~mm}$. The range of the upstream pressure $p_{\mathrm{i}}$ in each mixture composition and the corresponding upstream/downstream pressure ratio $p_{\mathrm{i}} / p_{\mathrm{o}}$ are listed with the mean Knudsen number range in Table II. These conditions were chosen to guaranty the mean Knudsen number values to be less than 0.08 . 
TABLE II. Measurement conditions for each mixture composition.

\begin{tabular}{cccccc}
\hline \hline Case & 1 & 2 & 3 & 4 & 5 \\
\hline $\mathrm{He}$ & $0 \%$ & $25 \%$ & $50 \%$ & $75 \%$ & $100 \%$ \\
$\mathrm{Ar}$ & $100 \%$ & $75 \%$ & $50 \%$ & $25 \%$ & $0 \%$ \\
\hline$p_{i}[\mathrm{kPa}]$ & $0.39-2.16$ & $0.46-1.91$ & $0.57-2.04$ & $0.76-2.08$ & $1.26-3.46$ \\
$p_{\mathrm{i}} / p_{\mathrm{o}}$ & $1.5-2.8$ & $1.5-2.7$ & $1.5-2.8$ & $1.5-2.7$ & $1.5-2.9$ \\
$K n_{\mathrm{m}}$ & $0.013-0.078$ & $0.016-0.078$ & $0.019-0.078$ & $0.025-0.077$ & $0.028-0.076$ \\
\hline \hline
\end{tabular}

For each mixture composition the mass flow rates were measured at four different mean Knudsen number ranges in the slip flow regime ${ }^{15)}$. This set of the measurements was repeated three times for each mixture (case 1 - 5) to check the repeatability of the measurement. The set of obtained mass flow rates was fitted according to eq. (6) via mean Knudsen number, and the viscous slip coefficient was calculated from the slope through the coefficient $A_{1}$ with eq. (2). The measurement error was evaluated in the same way as in Ref. 15. The extracted viscous slip coefficients for three measurements were averaged for every case, and the averaged results are listed in Table III. The obtained viscous slip coefficients for the mixtures are also plotted in Fig. 2 with filled black circles. From the figure, it is clearly shown that the viscous slip coefficient for the helium-argon binary mixture is a concave function of the molar fraction of the mixture, and the function is an asymmetric to the molar fraction. The peak value seems to appear at a little larger than $50 \%$ in the fraction of He, i.e. the fraction of He is a slightly larger than that of Ar.

The obtained here viscous slip coefficients are compared with the values measured by the spinning rotor gauge ${ }^{14)}$ plotted by open circles. The viscous slip coefficients obtained experimentally by two different methods show different behaviors both quantitatively and qualitatively. However, it was pointed out in Ref. 14 that the available experimental values show less satisfactory agreement with numerical results. Therefore, we also tried to compare our results with the numerically obtained viscous slip coefficients.

\section{B. Comparison with numerical simulations}

There are several numerical studies reported ${ }^{9-12)}$. Even though the numerical methods are different, they show similar results; thus, we only plot the results in the case of the complete accommodation at a surface; i.e. $\alpha_{\mathrm{He}}=\alpha_{\mathrm{Ar}}=1$, by Ivchenko et al. $(1997)^{9)}$ (listed in Table II of Sharipov and Kalempa $(2003)^{10)}$ ) by open triangles in Fig. 2. Although there is a quantitative discrepancy, the measured and numerical plots have the similar shape.

In the comparison with the numerical results, it is important to take into account the effect of the incomplete accommodation of molecules at a surface. As it was underlined previously, the viscous slip coefficient is a function of the tangential momentum accommodation coefficient (TMAC). The relation between the viscous slip coefficient $\sigma_{p}$ and TMAC 
$\alpha_{t}$ for single gas has been obtained by fitting the values of the viscosity slip coefficient calculated numerically for each value of the accommodation coefficient as $^{3)}$,

$$
\sigma_{p}=\frac{\sqrt{\pi}}{2} \frac{2-\alpha_{t}}{\alpha_{t}}\left(1+0.1366 \alpha_{t}\right)
$$

It is clear from eq. (8) that the viscous slip coefficient of a single gas is equal to 1.007 for $\alpha_{l}=1$ and it increases with decreasing TMAC from unity. Analyzing the experimental points of Fig. 2, one can see that the slip coefficient for the pure $\operatorname{Ar}(0 \%$ of $\mathrm{He})$ and pure $\mathrm{He}(100 \%$ of $\mathrm{He})$ are different from 1.007. From our measurements, TMAC for pure $\mathrm{He}$ and $\mathrm{Ar}$ is calculated by using eq. (8) to be $\alpha_{\mathrm{He}}=0.979 \pm 0.020, \alpha_{\mathrm{Ar}}=0.956 \pm 0.021$, respectively. The lighter gas species of He exhibits larger TMAC than the heavier $\mathrm{Ar}^{19)}$. The expression the analogous to eq. (8) is provided for the gas mixture, see eq. (20) in Ref. 9, as a function of TMAC for each component. However, the numerical calculations have to be done to obtain the explicit expressions of the viscosity slip coefficient for the mixture.

To investigate qualitatively the effect of the incomplete accommodation on the viscous slip coefficient for mixtures, TMAC is evaluated by comparing the measured viscous slip coefficient with those obtained numerically. We plot in Fig. 2 with open inverted triangles the numerical results obtained assuming the incomplete accommodation with separately measured values of $\alpha_{\mathrm{He}}=0.9542$ and $\alpha_{\mathrm{Ar}}=0.9105$ for each component ${ }^{12,9,14)}$ (there are no data for $\mathrm{He}=25 \%$ and $75 \%$ ). These results were reported by the same group as the results for the complete accommodation" shown in Fig. 2 with the open triangles. From Fig. 2, it is clear that the both numerical results show quite similar behaviors of the viscous slip coefficient as the function of molar fraction of He: the data seem to just shift upwards from the complete to the incomplete accommodation, namely decreasing TMAC. If we focus on the viscous slip coefficients for pure gas species, i.e. both ends of the plots, the increment from the complete to the incomplete accommodation is much larger for $\mathrm{He}=0 \%$ (pure Ar) compared with $\mathrm{He}=$ $100 \%$ (pure He). This is reasonable by the fact that $\alpha_{\mathrm{He}}>\alpha_{\mathrm{Ar}}$ in the numerical condition. Thus, the whole profile of the incomplete accommodation is not just shifting upwards but also slightly tilting to the right side from that of the complete accommodation. This tilting is coming from the difference of TMAC for each component; i.e. the positions of the plots at the both ends. Then, the profile of our measured viscous slip coefficients is compared with two numerical results with the complete and incomplete accommodation. The present experimental data exhibit a similar profile with the both numerical results, see Fig. 2; while, the curvature of the experimental data graph is much larger than those of two numerical ones, so the measured data intersect with the incomplete accommodation profile. The experimental TMACs for the pure gases $\left(\alpha_{\mathrm{He}}\right.$ $=0.979$ and $\left.\alpha_{\mathrm{Ar}}=0.956\right)$ are larger than that used in the numerical analysis $\left(\alpha_{\mathrm{He}}=0.9542\right.$ and $\left.\alpha_{\mathrm{Ar}}=0.9105\right)$; therefore, the 
experimental velocity slip coefficient is smaller than that of the incomplete accommodation numerical results for the pure gases. However, in the range of mole fraction of He between about $25-85 \%$, the experimental velocity slip coefficient is larger than that of the numerical incomplete accommodation, see Fig. 2. The corresponding TMACs for the mixture in that range have to be smaller than those used in numerical analysis with the incomplete accommodation. This is contradictory to the relation between the experimental and numerical TMACs for pure gases. Therefore, this result indicates that it is inappropriate to estimate the viscous slip coefficient of the mixture at any molar ratio numerically just by using TMAC for each component separately measured with a pure gas. If an "effective" TMAC for the mixture, which is expressed as a mean TMAC by a single value for a mixture, is assumed, it would vary with the molar fraction; it becomes smaller by mixing two components.

TABLE III. Viscous slip coefficient for mixture.

\begin{tabular}{cccccc}
\hline \hline case & 1 & 2 & 3 & 4 & 5 \\
\hline $\mathrm{He}$ & $0 \%$ & $25 \%$ & $50 \%$ & $75 \%$ & $100 \%$ \\
$\mathrm{Ar}$ & $100 \%$ & $75 \%$ & $50 \%$ & $25 \%$ & $0 \%$ \\
\hline$\sigma_{\mathrm{p}}$ & $1.094 \pm 0.024$ & $1.267 \pm 0.029$ & $1.391 \pm 0.033$ & $1.371 \pm 0.029$ & $1.048 \pm 0.021$ \\
\hline \hline
\end{tabular}

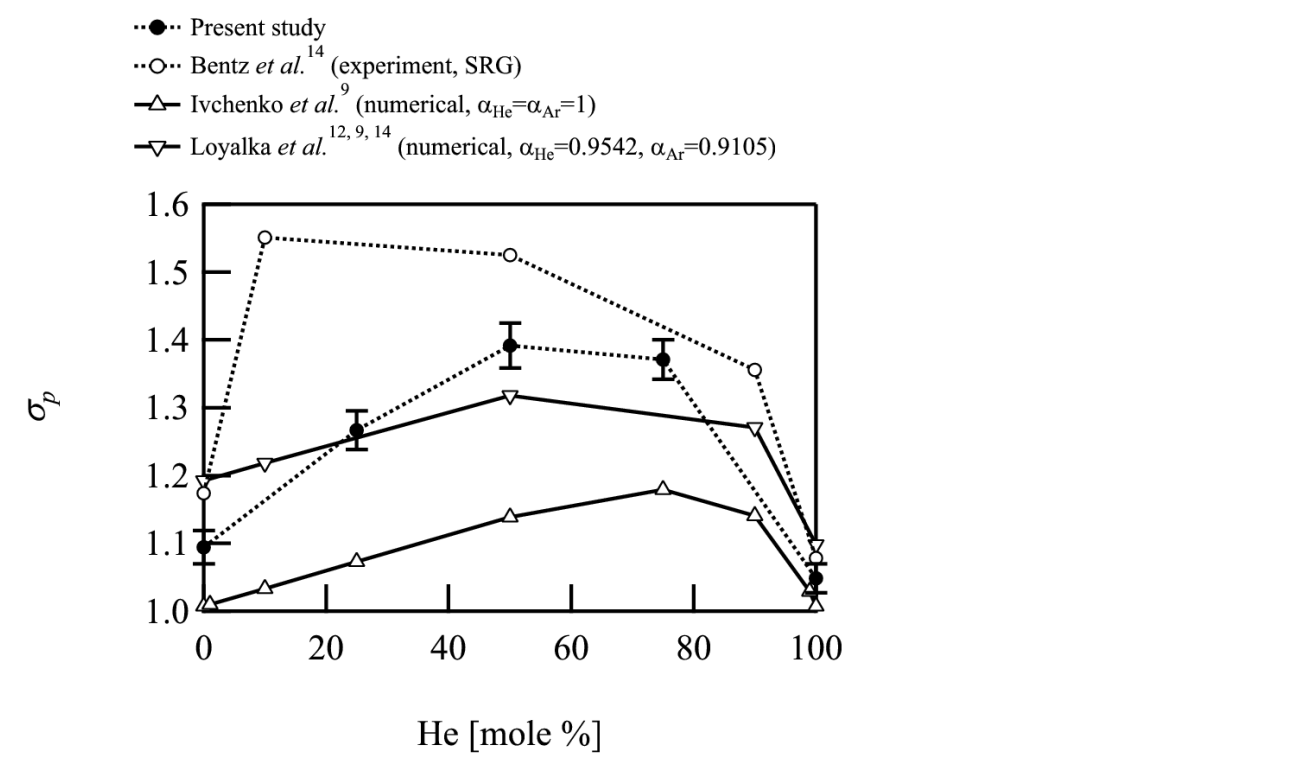

FIG. 2. The measured viscous slip coefficients for the mixtures are plotted by filled circles as a function of the molar fraction of He. They are compared with the experimental results measured by a spinning rotor gauge ${ }^{14)}$ plotted by open circles and the numerical results by assuming the complete accommodation ${ }^{9)}$ with open triangles and by assuming the incomplete accommodation ${ }^{12,9,14)}$ with open inverted triangles. 


\section{TIME VARIATION OF MIXTURE COMPOSITION THROUGH A MICROTUBE}

Since the viscous slip coefficients and TMACs for He and Ar are different, the volume flow rate through the microtube for each component could be different. In that case, the molar fraction of the downstream chamber varies with time from the originally supplied fraction. Thus, the time variation of the molar ratio in each case was measured at the downstream chamber by using QMS with the Multiple Concentration Detection (MCD) program in a software (Quadstar 32-bit, Pfeiffer, ver. 7.02). The gas in the downstream chamber was sampled continuously; therefore, the composition could not measure at the same time with the mass flow rate due to the change in pressure by the sampling. The measured time variation of the molar ratio cannot be compared directly with the measured mass flow rate in Section B, but it is still useful to know the characteristics of the flow of gaseous mixtures through a microtube. We note that the continuous sampling is achieved with a variable leak valve, which could be considered as a slit. This slit is necessary to limit the flow to the mass spectrometer, while not to change too much the downstream pressure. We assume that this variable leak valve do not modify significantly the molar ratio. Adding to that, the sampling flow rate is controlled manually so that the quantitative comparison between measurements is also not adequate.

The time variation of the molar ratio was measured at the mean Knudsen numbers equal to the minimum $\left(K n_{\mathrm{m}} \sim 0.02\right)$ and maximum $\left(K n_{\mathrm{m}} \sim 0.08\right)$ for each measurement for each mixture composition (Section III). The time constants to reach equilibrium were different in each measurement, so the molar ratios for the first 1 hour are plotted in Fig. 3. From the figure it is clear that, Ar molar fraction increases, so the He molar fraction decreases with time in every cases. Thus, the volume flow rate of Ar could be larger than that of He; i.e. Ar flows much faster than He. This result looks contradictory to the numerical result ${ }^{20)}$ which showed that lighter component has the higher velocity for the rarefaction parameter, which is inversely proportional to the Knudsen number, varying from 0.1 to 40 . This discrepancy between the experimental and numerical results would be explained by the effect of TMAC. In the above mentioned numerical study, the diffuse scattering, $\alpha_{\mathrm{He}}=\alpha_{\mathrm{Ar}}=1$, was assumed at a surface. Therefore, the viscous slip coefficient, which is directly related to the volume flow rate, were the same for $\mathrm{He}$ and Ar there. Adding to that our measurements were carried out in the slip flow regime where $K n_{\mathrm{m}}$ $<0.1$, the difference in the macroscopic velocity of each component under the complete accommodation could be small ${ }^{20)}$. Meanwhile, considering TMACs of each species obtained in our measurements $\left(\alpha_{\mathrm{He}}=0.979, \alpha_{\mathrm{Ar}}=0.956\right)$, the viscous slip coefficient of each component is larger than that of the case of full accommodation (Fig. 2). In addition, the viscous slip coefficient of Ar has larger value than that of He (Table 1). This difference in the viscous slip coefficients for two components could overcome the difference under the complete accommodation and lead to the larger volume flow rate for 
Ar. It is important to note that we cannot conclude on the relation between the velocity and the molecular weights of components because the volume flow rate is dependent on TMAC or the viscous slip coefficient of each component on the surface employed.

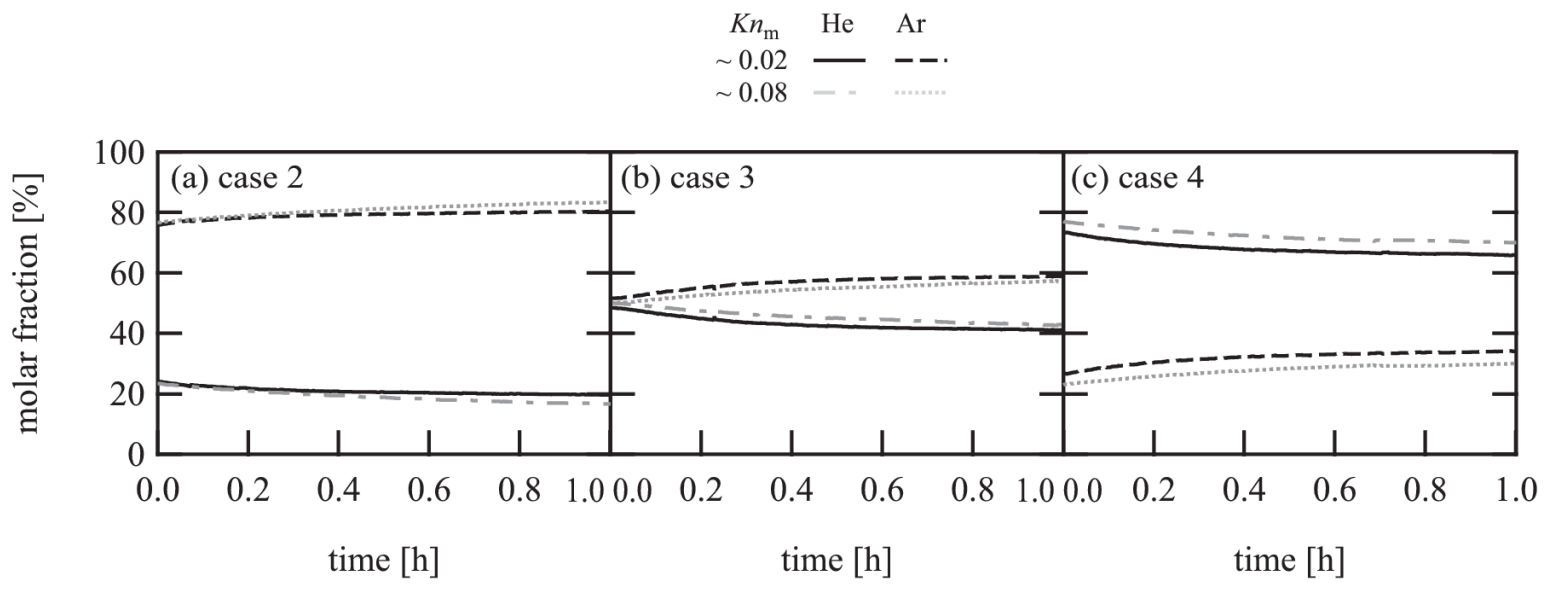

FIG. 3. The time variation of the molar fraction at the downstream chamber for each case are plotted up to $1 \mathrm{~h}$ : (a) case 2 (He, Ar) $=(25 \%$, $75 \%)$, (b) case $3(\mathrm{He}, \mathrm{Ar})=(50 \%, 50 \%)$ and (c) case $4(\mathrm{He}, \mathrm{Ar})=(75 \%, 25 \%)$. The profiles are drawn with smaller mean Knudsen number for He by black solid lines and for Ar by black dashed lines, with larger mean Knudsen number for He by gray dashed-dotted lines and for Ar by gray dotted lines, respectively.

\section{CONCLUSION}

The mass flow rate through a single microtube for helium-argon binary gas mixture was measured by the constantvolume method, and the viscous slip coefficient was obtained by fitting the measured mass flow rate with an analytical expression as a function of the mean Knudsen number. The measurement was limited in the slip flow regime where the mean Knudsen number was less than 0.1 . The viscous slip coefficients of binary gas mixtures exhibit a concave function of the molar ratio of the mixture. Although there is a discrepancy between the measured viscous slip coefficients and the reported values measured by a spinning rotor gauge, the tendency of the profile in the present study is similar to the numerical results with the complete accommodation. To investigate the effect of the tangential momentum accommodation coefficient, the measured results were compared with the two numerical results assuming the complete and incomplete accommodation at a surface. The relation between the measured and numerical viscous slip coefficient varies depending on the molar ratio of the mixture. Thus, it is inappropriate to estimate numerically the viscous slip coefficient of the mixture at any certain molar ratio from the tangential momentum accommodation coefficient of a pure gas for each component. The time variation of the molar ratio through a microtube was measure by sampling the gas in the downstream chamber. The molar ratio of helium decreases 
with time, indicating the volume flow rate of argon is larger than that of helium. Although this is not consistent with the velocity profiles obtained numerically assuming the complete accommodation, this difference could be explained by the difference in the tangential momentum accommodation coefficient between helium and argon.

\section{ACKNOWLEDGMENTS}

This work was partially supported by JSPS KAKENHI Grant Number 26630049.

\section{REFERENCES}

${ }^{1}$ F. Sharipov and V. Seleznev, "Data on Internal Rarefied Gas Flows," J. Phys. Chem. Ref. Data 27, 657 (1998).

${ }^{2}$ A. Agrawal and S. V. Prabhu, "Survey on measurement of tangential momentum accommodation coefficient," J. Vac. Sci. Technol. A 26, 634 (2008).

${ }^{3}$ F. Sharipov, "Data on the Velocity Slip and Temperature Jump on a Gas-Solid Interface," J. Phys. Chem. Ref. Data 40, $023101(2011)$.

${ }^{4}$ A. Agrawal, "A Comprehensive Review on Gas Flow in Microchannels," Int. J. Micronano Scale Transp. 2, 1 (2011).

${ }^{5}$ F. Sharipov, "Gaseous mixtures in vacuum systems and microfluidics," J. Vac. Sci. Technol. 31(5), 050806 (2013).

${ }^{6}$ F.O. Goodman and H. Y. Wachman, Dynamics of Gas-Surface Scattering, (Academic, New York, 1976).

${ }^{7}$ I. A. Graur, J. G. Méolans and D. E. Zeitoun, "Analytical and numerical description for isothermal gas flows in microchannels," Microfluid. Nanofluid. 2, 64 (2006).

${ }^{8}$ G. A. Bird, Molecular gas dynamics and the direct simulation of gas flows, (Clarendon, Oxford, 1994).

${ }^{9}$ I.V. Ivchenko, S.K. Loyalka and R. V. Tompson, "Slip coefficients for binary gas mixtures," J. Vac. Sci. Technol. A 15, 2375 (1997).

${ }^{10}$ F. Sharipov and D. Kalempa, "Velocity slip and temperature jump coefficients for gaseous mixtures. I. Viscous slip coefficient," Phys. Fluids 15, 1800 (2003).

${ }^{11}$ C. E. Siewert and D. Valougeorgis, "Concise and accurate solutions to half-space binary-gas flow problems defined by the McCormack model and specular-diffuse wall conditions," Eur. J. Mech. B Fluids 23, 709 (2004).

${ }^{12}$ S. K. Loyalka, "Velocity Slip Coefficient and the Diffusion Slip Velocity for a Multicomponent Gas Mixture," Phys. Fluids $14,2599(1971)$.

${ }^{13}$ J. Pitakarnnop, S. Varoutis, D. Valougeorgis, S. Geoffroy, L. Baldas and S. Colin, "A novel experimental setup for gas microflows," Microfluid. Nanofluid. 8, 57 (2010). 
${ }^{14}$ J. A. Bentz, R. V. Tompson and S. K. Loyalka, "Viscosity and velocity slip coefficients for gas mixtures: Measurements with a spinning rotor gauge," J. Vac. Sci. Technol. A 17, 236 (1999).

${ }^{15}$ H. Yamaguchi, T. Hanawa, O. Yamamoto, Y. Matsuda, Y. Egami and T. Niimi, "Experimental measurement on tangential momentum accommodation coefficient in a single microtube," Microfluid. Nanofluid. 11, 57 (2011).

${ }^{16}$ T. Ewart, P. Perrier, I. A. Graur and J. G. Méolans, "Mass flow rate measurements in a microchannel, from hydrodynamic to near free molecular regimes," J. Fluid. Mech. 584, 337 (2007).

${ }^{17}$ E. B. Arkilic, K. S. Breuer and M. A. Schmidt, "Mass flow and tangential momentum accommodation in silicon micromachined channels," J. Fluid. Mech. 437, 29 (2001).

${ }^{18}$ R. C. Reid, J. M. Prausnitz and T. K. Sherwood, The Properties of Gases and Liquids, Third. Edition (McGraw-Hill, New York, 1977).

${ }^{19}$ P. Perrier, I. A. Graur, T. Ewart and J. G. Méolans, " Mass flow rate measurements in microtubes: From hydrodynamic to near free molecular regime," Phys. Fluids 23, 042004 (2011).

${ }^{20}$ F. Sharipov and D. Kalempa, "Gaseous mixture flow through a long tube at arbitrary Knudsen numbers," J. Vac. Sci. Technol. A 20, 814 (2002). 